\title{
CSSs in a sample of B2 radio sources of intermediate strength
}

\author{
D. J. Saikia ${ }^{1,2}$, P. Thomasson ${ }^{2}$, R. E. Spencer ${ }^{2}$, F. Mantovani ${ }^{3}$, C. J. Salter ${ }^{4}$, and S. Jeyakumar ${ }^{5}$ \\ 1 National Centre for Radio Astrophysics, Tata Institute of Fundamental Research, Post Bag No. 3, Ganeshkhind, \\ Pune 411 007, India, \\ 2 Jodrell Bank Observatory, University of Manchester, Macclesfield, Cheshire SK11 9DL, UK \\ 3 Istituto di Radioastronomia, CNR, via P. Gobetti 101, Bologna, Italy \\ 4 Arecibo Observatory, HC3 Box 53995, Arecibo, Puerto Rico PR 00612, USA \\ 5 Physikalisches Institut, Universität zu Köln, Zülpicher Str. 77, 50937 Köln, Germany
}

Received 12 March 2002 / Accepted 28 May 2002

\begin{abstract}
We present radio observations of 19 candidate compact steep-spectrum (CSS) objects selected from a well-defined, complete sample of $52 \mathrm{~B} 2$ radio sources of intermediate strength. These observations were made with the VLA A-array at 4.835 GHz. The radio structures of the entire sample are summarised and the brightness asymmetries within the compact sources are compared with those of the more extended ones, as well as with those in the 3CRR sample and the CSSs from the B3-VLA sample. About 25 per cent of the CSS sources exhibit large brightness asymmetries, with a flux density ratio for the opposing lobes of $>5$, possibly due to interaction of the jets with infalling material. The corresponding percentage for the larger-sized objects is only about 5 per cent. We also investigate a possible dependence of the flux density asymmetry of the lobes on redshift, since this might be affected by more interactions and mergers in the past. No such dependence is found. A few individual objects of interest are discussed in the paper.
\end{abstract}

Key words. galaxies: active - quasars: general - galaxies: nuclei - radio continuum: galaxies

\section{Introduction}

Statistical studies of the structures of radio sources have played an important role in understanding both their cosmological evolution and their evolution with age. One of the best studied complete samples is the 3CRR which has complete identification and redshift information (Laing et al. 1983). Examples of other well-studied samples covering significant areas of the sky are the Molonglo Reference Catalogue 1-Jy sample (Large et al. 1981; Kapahi et al. 1998a,b), the 2-Jy all-sky (Wall \& Peacock 1985 and references therein) and the S4 samples (Pauliny-Toth et al. 1978; Kapahi 1981; Stickel \& Kühr 1994; Saikia et al. 2001). Studies of compact steep spectrum sources (CSSs) in such well-defined source samples have provided useful insights towards understanding the evolution of radio sources (cf. O'Dea 1998 for a review). In recent years, attention has also been focussed on CSSs selected from samples that are significantly weaker than those investigated earlier (e.g. Fanti et al. 2001; Kunert et al. 2002).

The CSSs are physically small objects of subgalactic dimensions. They are believed to be young sources at an early stage of evolution (Fanti et al. 1995; Readhead et al. 1996a,b; O’Dea 1998; Owsianik \& Conway 1998). Possibly fuelled by

Send offprint requests to: D. J. Saikia,

e-mail:djs@ncra.tifr.res.in the infall of gas, their sizes and structures appear to be affected by the ambient gas in the central regions of the host galaxies. At sub-arcsec resolution, these sources often show strongly distorted structures with recognizable jet-like features (e.g. Spencer et al. 1991; Schilizzi et al. 2000), suggesting strong dynamical interaction with the external medium. Evidence for the presence of this gas is also seen in polarization observations of CSSs, which are generally weakly polarized ( $\leq 1$ per cent) at or below about $5 \mathrm{GHz}$ (Saikia et al. 1985, 1987). The increase in the median polarization with frequency (van Breugel et al. 1992; Akujor \& Garrington 1995) suggests that the depolarization is due to the Faraday effect. A number of CSSs have also been found to exhibit large rotation measures, leading Mantovani et al. (1994) to suggest that most CSSs may be cocooned in dense gaseous envelopes. The radio structures of CSSs tend to be more asymmetric than is the case for the larger sources (Saikia et al. 1995; Jeyakumar \& Saikia 2000; Saikia et al. 2001), indicating an asymmetry in the distribution of gas on opposite sides of the nucleus on these scales. Evidence for such asymmetries in the gas distribution, which might be related to the fuelling of the radio source, is sometimes provided through a huge difference between the rotation measures on opposite sides of the nucleus (Mantovani et al. 1994; Junor et al. 1999). In addition to the ionized component, there is also evidence for a high incidence of associated HI 


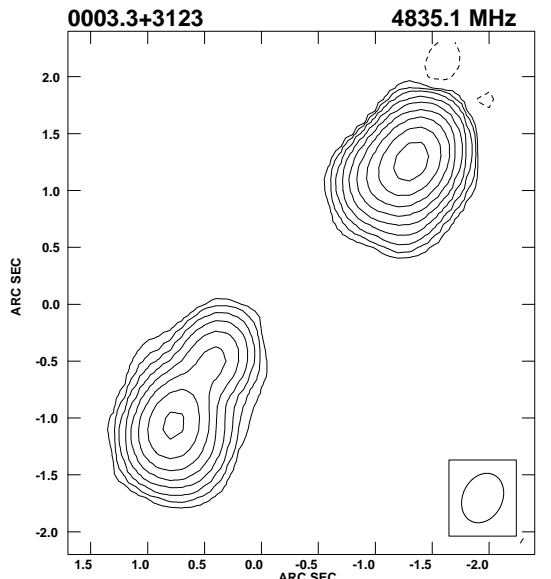

Peak: 1.0706E-01 Cont: $3.000 \mathrm{E}-04$
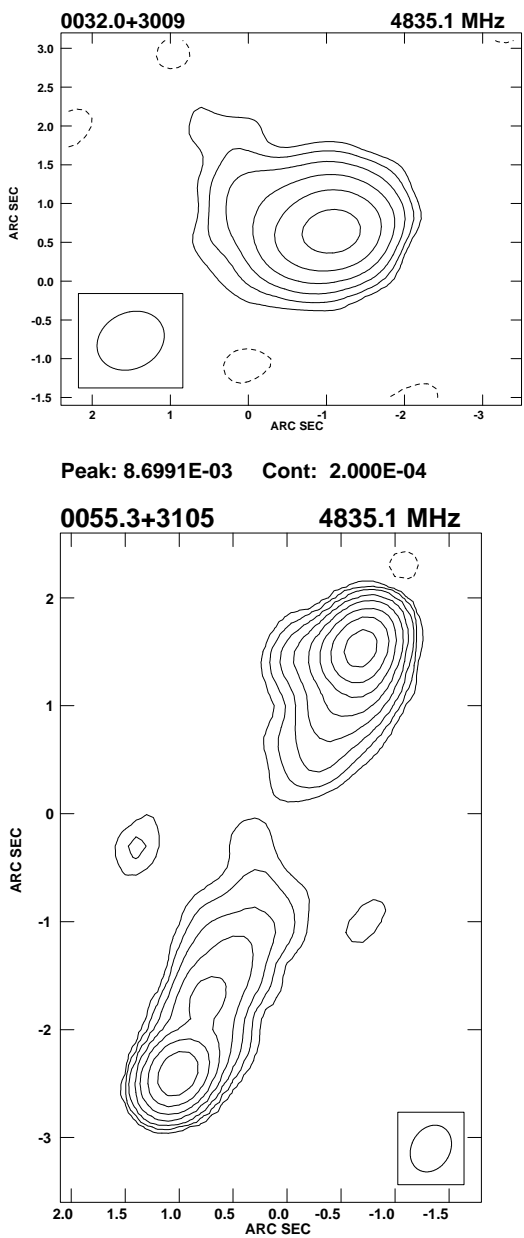

Peak: $5.5175 \mathrm{E}-02$ Cont: $3.000 \mathrm{E}-04$

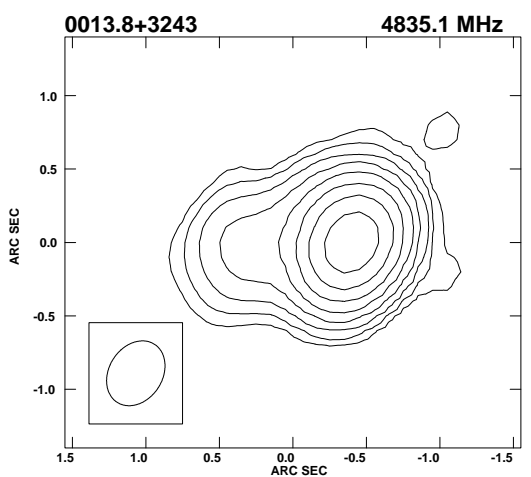

Peak: 4.2021E-02 Cont: $2.000 \mathrm{E}-04$

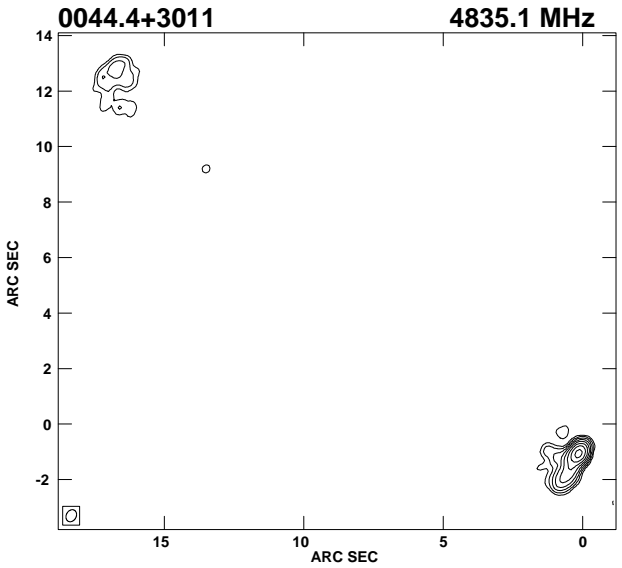

Peak: 4.8408E-02 Cont: $3.000 \mathrm{E}-04$

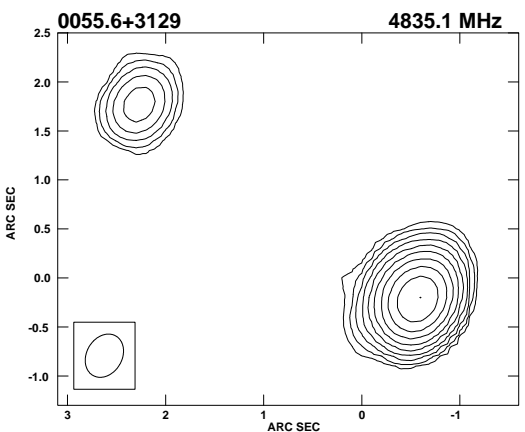

Peak: 1.0286E-01 Cont: 2.000E-04

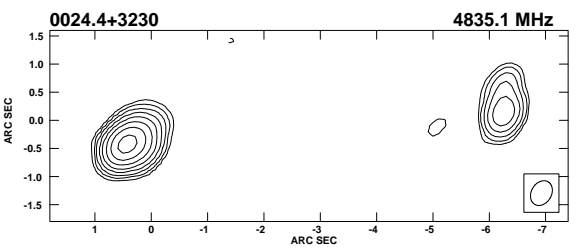

Peak: 3.5287E-02 Cont: $2.000 \mathrm{E}-04$

$0051.5+3144 \quad 4835.1 \mathrm{MHz}$
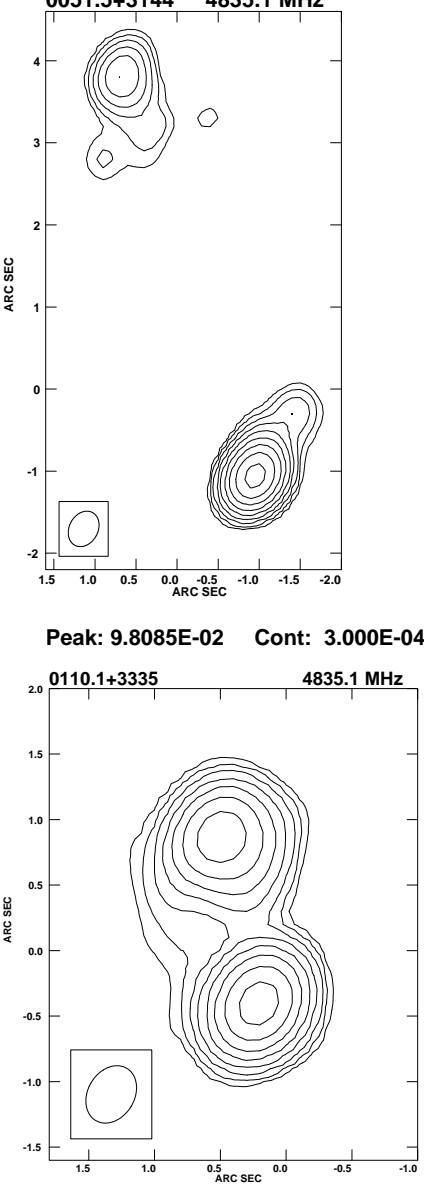

Peak: 3.8290E-02 Cont: 2.000E-04

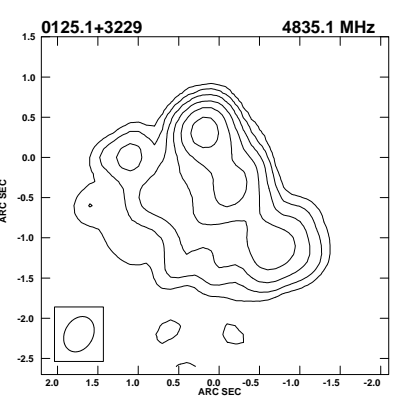

Peak: 1.4307E-02 Cont: $3.000 \mathrm{E}-04$

Fig. 1. VLA images of our sample of sources. The contour levels for all the images are $-1,1,2,4,8,16, \ldots$ times the first contour level. The peak brightness in the image and the level of the first contour in units of Jy/beam are given below the images. The half-power ellipse of the restoring beam is shown in one of the lower corners of each image.

absorption towards CSSs (Carilli et al. 1998; Peck et al. 2000; Morganti et al. 2001).

In this paper, we study the small but well-defined sample of sources of intermediate strength described in Sect. 2, and concentrate on the radio structures of CSSs in this sample. The radio observations and the results from these observations are presented in Sect. 3. The discussion and concluding remarks are presented in Sects. 4 and 5 respectively. 


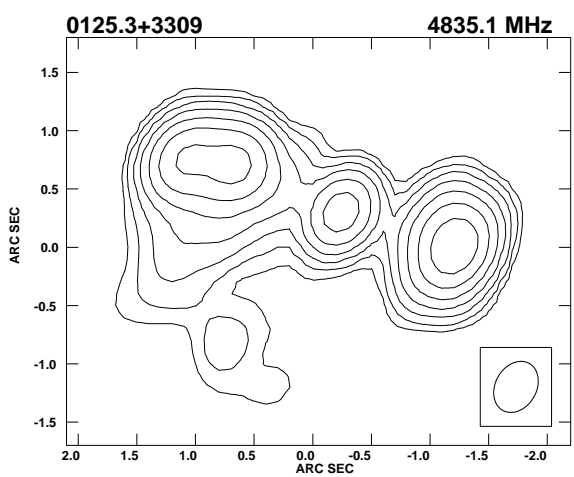

Peak: 5.8456E-02 Cont: 5.000E-04
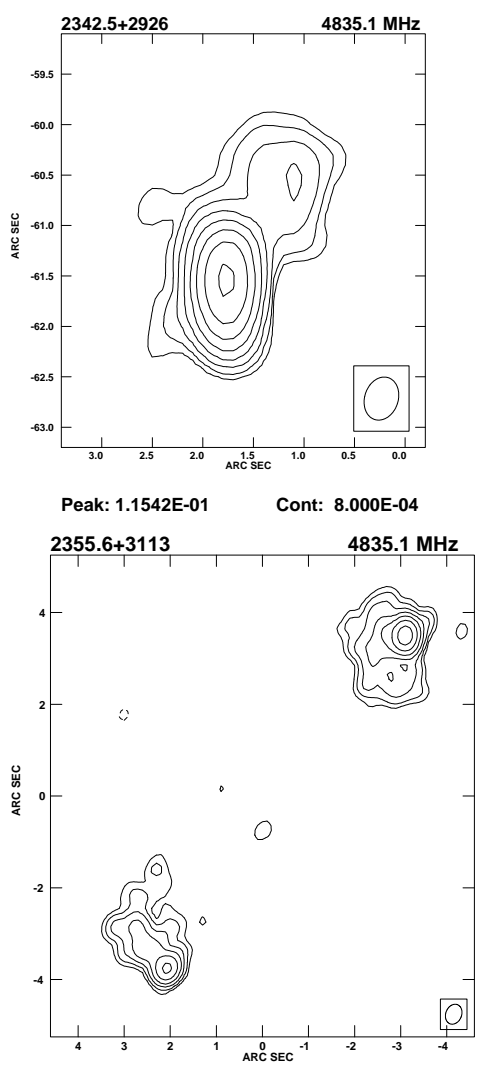

Peak: 1.9859E-02 Cont: 2.000E-04

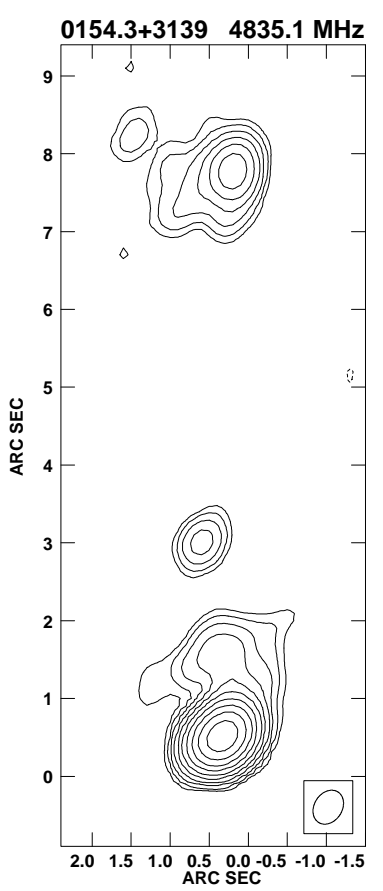

Pk:2.6E-01 Cnt:3.0E-04

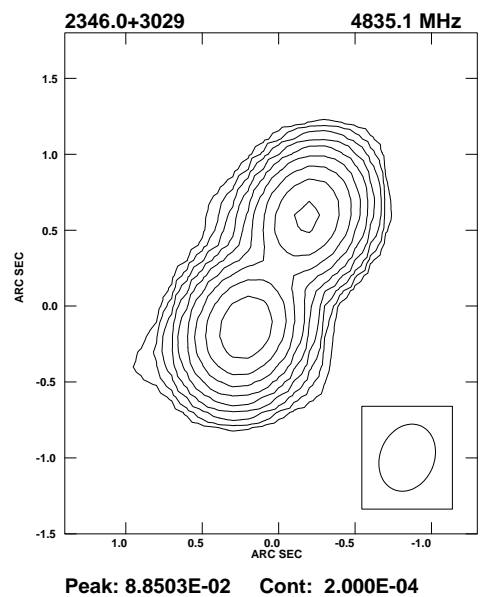

Peak: $8.8503 \mathrm{E}-02$ Cont: $2.000 \mathrm{E}-04$

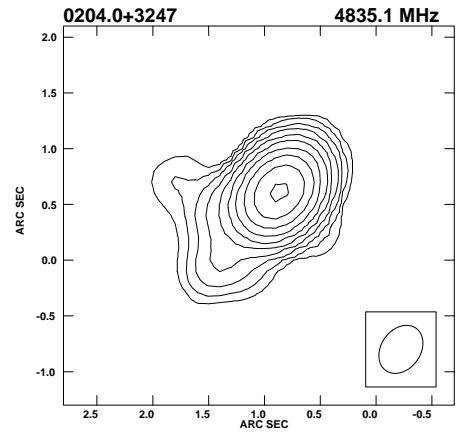

Peak: 2.3164E-01 Cont: $4.000 \mathrm{E}-04$

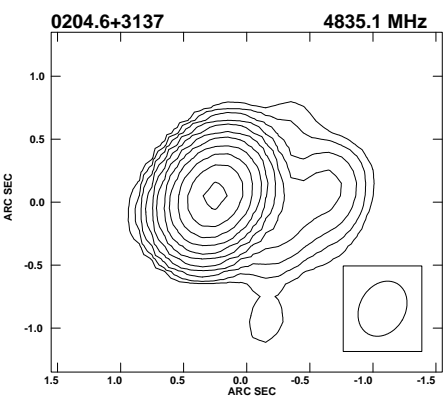

Peak: 4.9131E-01 Cont: 4.000E-04

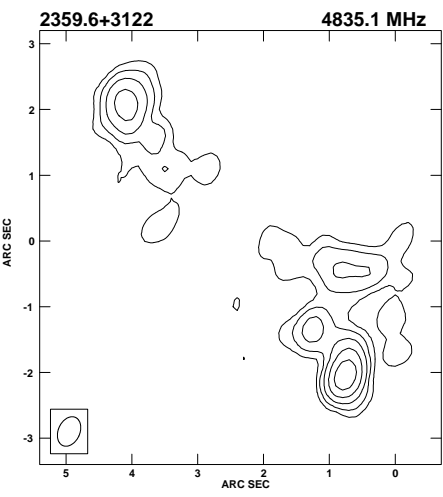

Peak: 1.0491E-02 Cont: 4.000E-04

Fig. 2. See the caption to Fig. 1.

\section{The B2 intermediate-strength sample}

The sample of sources of intermediate strength selected from the 408-MHz Bologna B2.1 catalogue (Colla et al. 1970) consists of 52 objects which satisfy the following criteria. They lie within the region, $23^{\mathrm{h}} 30^{\mathrm{m}}<\mathrm{RA}(\mathrm{B} 1950)<2^{\mathrm{h}} 30^{\mathrm{m}}$ and $29^{\circ} 18^{\prime}<$ $\operatorname{Dec}(\mathrm{B} 1950)<34^{\circ} 02^{\prime}$, and their flux densities are in the range $0.9 \leq \mathrm{S}_{408}<2.5 \mathrm{Jy}$. These sources have been observed earlier with the Mk IA - Defford interferometer at $408 \mathrm{MHz}$ (Warwick 1977), and the Westerbork Synthesis Radio Telescope (WSRT) at $5 \mathrm{GHz}$ (Padrielli et al. 1981, hereinafter referred to as P81). Optical identifications and redshift measurements are not available for most of the sources at present, although the predicted median redshift for a $408-\mathrm{MHz} 1-\mathrm{Jy}$ sample for a range of models is in very close agreement and yields a value of about 1.2 (cf. Dunlop \& Peacock 1990).

The radio structures of many of the sources in this sample, especially the smaller ones, were not clear from the earlier observations. The main objective of the present project is to investigate the structure and properties of CSSs of intermediate strength, and compare these with the larger sources using a well-defined source sample selected at a low frequency.

\section{Observations, analysis and results}

Eighteen steep-spectrum sources $\left(\alpha_{400}^{5000} \gtrsim 0.5\right.$ (P81), where $S \propto v^{-\alpha}$ ) from the B2 sample of intermediate strength were observed in snapshot mode with the Very Large Array (VLA) 
Table 1. The observational parameters and derived properties for the sources observed.

\begin{tabular}{|c|c|c|c|c|c|c|c|c|c|c|c|c|c|c|}
\hline Source & Opt. & $z$ & " & $\begin{array}{c}\mathrm{am} \text { siz } \\
\quad \prime\end{array}$ & $\circ$ & $\begin{array}{r}\mathrm{rms} \\
\mu \mathrm{Jy} / \mathrm{b}\end{array}$ & Cmp. & $\begin{array}{r}S_{\mathrm{p}} \\
\mathrm{mJy} / \mathrm{b}\end{array}$ & $\begin{array}{c}S_{\text {int }} \\
\text { mJy }\end{array}$ & $\begin{array}{r}S_{\mathrm{t}} \\
\mathrm{mJy}\end{array}$ & Str. & FR & $\begin{array}{r}\text { LAS } \\
\prime \prime\end{array}$ & Notes \\
\hline $0003.3+3123$ & & & $\overline{0.45}$ & 0.35 & 154 & 91 & $\mathrm{~N}$ & 107 & 146 & 223 & $\bar{D}$ & II & 3.2 & \\
\hline & & & & & & & S & 45 & 77 & & & & & \\
\hline $0013.8+3243$ & & & 0.47 & 0.36 & 147 & 50 & RS & 42 & 57 & 57 & $\mathrm{D}$ ? & & 0.6 & \\
\hline $0024.4+3230$ & & & 0.47 & 0.36 & 149 & 60 & $\begin{array}{c}\text { W } \\
\text { E }\end{array}$ & $\begin{array}{r}5.6 \\
35\end{array}$ & $\begin{array}{r}8.7 \\
52\end{array}$ & 61 & $\mathrm{D}$ & II & 6.7 & \\
\hline $0032.0+3009$ & G & 0.1744 & 0.89 & 0.72 & 117 & 80 & $\mathrm{RS}$ & 8.7 & 15 & 15 & SR & & $\sim 1$ & \\
\hline $0044.4+3011$ & & & 0.45 & 0.35 & 151 & 64 & $\begin{array}{c}\text { W } \\
\text { E }\end{array}$ & $\begin{array}{r}48 \\
1.9\end{array}$ & $\begin{array}{l}88 \\
12\end{array}$ & 101 & $\mathrm{D}$ & II & 22 & \\
\hline $0051.5+3144$ & & & 0.45 & 0.35 & 152 & 83 & $\begin{array}{l}\mathrm{N} \\
\mathrm{S}\end{array}$ & $\begin{array}{r}9.6 \\
98\end{array}$ & $\begin{array}{r}14 \\
121\end{array}$ & 137 & D & II & 5.1 & \\
\hline $0055.3+3105$ & & & 0.45 & 0.36 & 151 & 82 & $\begin{array}{l}\mathrm{N} \\
\mathrm{S}\end{array}$ & $\begin{array}{l}55 \\
35\end{array}$ & $\begin{array}{l}82 \\
57\end{array}$ & 140 & $\mathrm{D}$ & II & 4.3 & \\
\hline $0055.6+3129$ & G & & 0.47 & 0.36 & 150 & 67 & $\begin{array}{l}\text { W } \\
\text { E }\end{array}$ & $\begin{array}{r}103 \\
4.8\end{array}$ & $\begin{array}{r}119 \\
5.5\end{array}$ & 125 & D & II & 3.5 & \\
\hline $0110.1+3335$ & & & 0.46 & 0.35 & 149 & 62 & $\begin{array}{l}\mathrm{N} \\
\mathrm{S}\end{array}$ & $\begin{array}{l}21 \\
38\end{array}$ & $\begin{array}{l}29 \\
43\end{array}$ & 72 & D & II & 1.3 & \\
\hline $0125.1+3229$ & & & 0.46 & 0.35 & 151 & 84 & RS & 14 & 64 & 64 & $?$ & & 2.1 & $\mathrm{n}$ \\
\hline $0125.3+3309$ & & & 0.46 & 0.35 & 151 & 148 & $\begin{array}{l}\text { W } \\
\text { C } \\
\text { E }\end{array}$ & $\begin{array}{l}58 \\
24 \\
45\end{array}$ & $\begin{array}{r}86 \\
28 \\
119\end{array}$ & 234 & $\mathrm{~T}$ & II & 2.3 & \\
\hline $0154.3+3139$ & Q & 0.373 & 0.46 & 0.36 & 148 & 84 & $\begin{array}{l}\mathrm{N} \\
\mathrm{C} \\
\mathrm{S}\end{array}$ & $\begin{array}{r}16 \\
3.6 \\
260\end{array}$ & $\begin{array}{r}30 \\
3.6 \\
338\end{array}$ & 371 & $\mathrm{~T}$ & II & 7.3 & \\
\hline $0204.0+3247$ & & & 0.47 & 0.35 & 144 & 69 & RS & 232 & 260 & 260 & $\mathrm{D} ?$ & & 0.8 & \\
\hline $0204.6+3137$ & G & 1.213 & 0.46 & 0.36 & 150 & 158 & RS & 491 & 507 & 503 & CJ? & & 0.9 & $\mathrm{n}$ \\
\hline $2330.5+2937$ & & & 0.46 & 0.35 & 160 & 40 & $\mathrm{RS}$ & 81 & 82 & 82 & $\mathrm{U}$ & & $<0.1$ & $\mathrm{n}$ \\
\hline $2342.5+2926$ & G & 0.13069 & 0.44 & 0.33 & 162 & 104 & $\begin{array}{l}\mathrm{N} \\
\mathrm{S}\end{array}$ & $\begin{array}{r}7.0 \\
115\end{array}$ & $\begin{array}{r}13 \\
239\end{array}$ & 263 & $\mathrm{D}$ & II? & 1.2 & $\mathrm{n}$ \\
\hline $2346.0+3029$ & & & 0.45 & 0.35 & 158 & 50 & $\begin{array}{l}\mathrm{N} \\
\mathrm{S}\end{array}$ & $\begin{array}{l}60 \\
89\end{array}$ & $\begin{array}{l}64 \\
91\end{array}$ & 160 & D & II & 0.8 & \\
\hline $2355.6+3113$ & & & 0.45 & 0.34 & 158 & 48 & $\begin{array}{l}\mathrm{N} \\
\mathrm{S}\end{array}$ & $\begin{array}{r}20 \\
7.6\end{array}$ & $\begin{array}{l}46 \\
23\end{array}$ & 70 & D & II & 8.9 & \\
\hline $2359.6+3122$ & & & 0.47 & 0.33 & 156 & 86 & $\begin{array}{l}\text { W } \\
\text { E }\end{array}$ & $\begin{array}{r}10 \\
4.5\end{array}$ & $\begin{array}{l}35 \\
18\end{array}$ & 62 & D & II & 5.3 & \\
\hline
\end{tabular}

A-array at $4835 \mathrm{MHz}$ on 1985 February 10. All of these were either unresolved or only slightly resolved by the WSRT 5-GHz observations of P81 made with an angular resolution of about $6 \times 6 \operatorname{cosec} \delta \operatorname{arcsec}^{2}$, where $\delta$ is the declination of the source. In addition to these 18 , the sample source $0204.6+3137$ which was listed by P81 as having a spectral index $\alpha_{408}^{5000}=0.47$ was also observed.

The data were calibrated at the VLA and reduced using standard imaging and CLEANing procedures in the AIPS (Astronomical Image Processing System) package. All flux densities are on the Baars et al. (1977) scale.

The VLA radio images for the 18 resolved objects are presented in Figs. 1 and 2. The observational parameters, and some of the properties derived from the images, are listed for all 19 sources in Table 1, which is arranged as follows. Column 1: source name in the IAU format using the B1950 coordinates; Col. 2: optical classification for those objects with confirmed identifications, where $\mathrm{G}$ denotes a galaxy and $\mathrm{Q}$ a quasar; Col. 3: redshift; Col. 4: the major and minor axes of the restoring beam in arcsec and its position angle (PA) in degrees; Col. 5: the rms noise in the total-intensity images in units of $\mu \mathrm{Jy} / \mathrm{beam}$; Col. 6: component designations for the structures revealed by the VLA images, with RS denoting the entire radio source for those without well-defined doublelobed structure; Cols. 7 and 8: the peak and total flux density of the components in units of mJy/beam and mJy; Col. 9: the total flux density of the source in units of $\mathrm{mJy}$ determined by integrating over a box containing all emission detected in the VLA images; Col. 10: the classification of the radio structure of the source as determined from our images or those available in the literature. Here $\mathrm{D}$ denotes a double, $\mathrm{T}$ a triple, $\mathrm{CJ}$ a core-jet source, HT a head-tail source, $\mathrm{C}+\mathrm{E}$ a core with some extension, while $\mathrm{SR}$ is a slightly resolved source and $\mathrm{U}$ an unresolved source. Column 11: the Fanaroff-Riley classification for double-lobed and triple sources based on an examination of the radio image. A question mark (?) in Cols. 10 or 11 denotes an uncertainty in the classification. Column 12: the largest angular size (LAS) of the source in arcsec. These are usually measured from the outermost peaks of radio emission. Column 13: Here $\mathrm{n}$ denotes that there is a note on the source in Sect. 3.1 of this paper. 

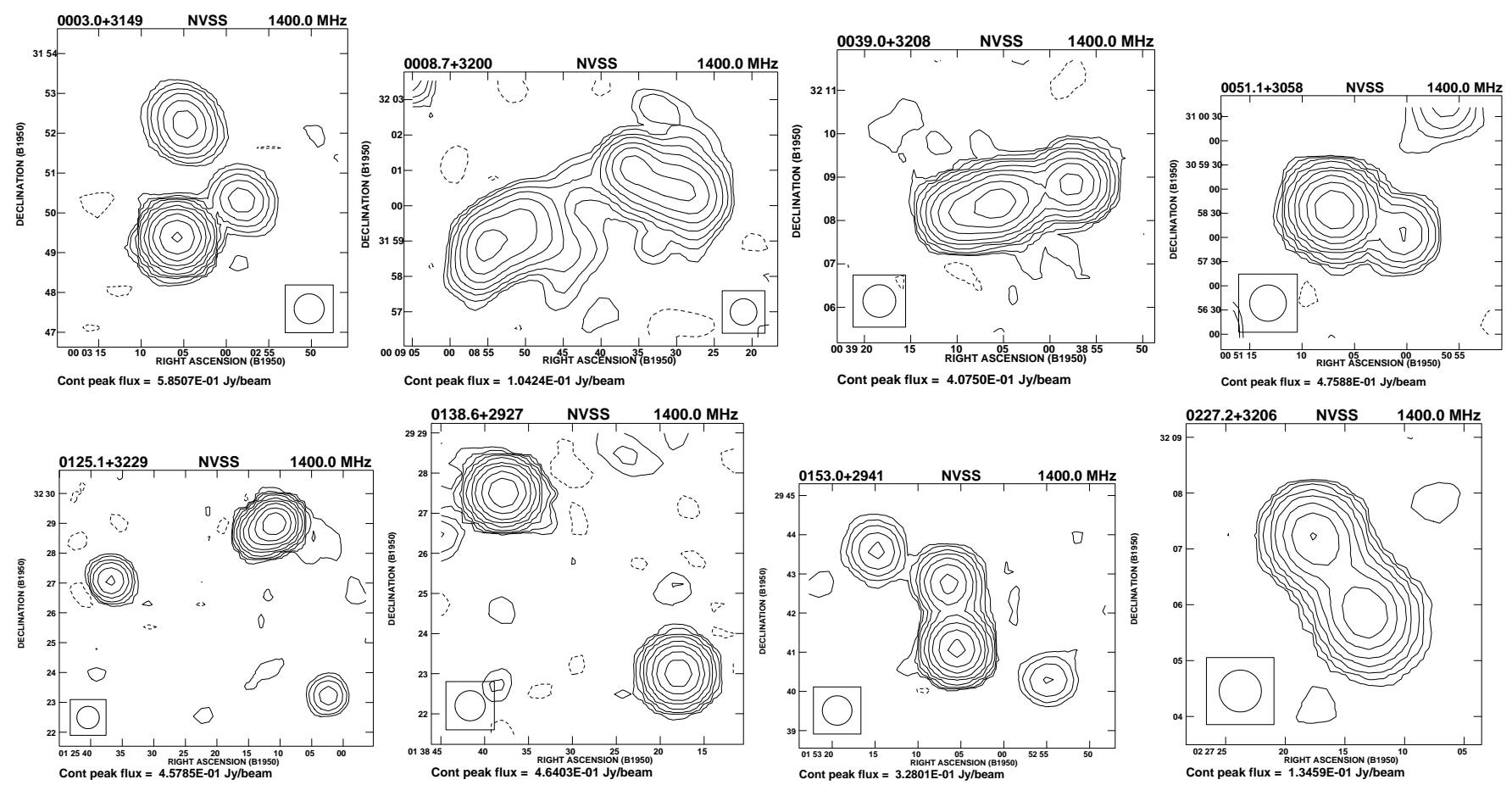

Fig. 3. NVSS images of the sources discussed in the text. The contour levels for all the images are $-1,1,2,4,8,16, \ldots$ mJy/beam. The peak brightness in units of Jy/beam is given below each image.

For the 33 sample sources not observed by us, Table 2 lists the source name, optical identification, redshift, structural classification, Fanaroff-Riley class, largest angular size and references. The above parameters have the same meaning as in Table 1. The sources for which the LAS has been estimated from the NVSS (Condon et al. 1998) images have a superscript $\mathrm{N}$ in Col. 6.

\subsection{Notes on individual sources}

Eight images from the NVSS survey (Condon et al. 1998) are presented in Fig. 3 as these provide significant extra information on sample sources, both those observed and not observed by us.

0003.0+3149 The NVSS image shows three non-collinear components (Fig. 3). It is not clear whether they are all related. Using the WENSS (Rengelink et al. 1997) and NVSS (Condon et al. 1998) flux densities, the spectral index of the dominant component is $\alpha_{327}^{1400} \sim 0.40$ while that of the two weaker components in order of increasing right ascension are $\sim 0.87$ and 0.82 respectively.

$\mathbf{0 0 0 8 . 7 + 3 2 0 0}$ The position of the optical galaxy at RA(B1950) $00^{\mathrm{h}} 08^{\mathrm{m}} 43^{\mathrm{s}} 6$ and $\operatorname{Dec}(\mathrm{B} 1950) 32^{\circ} 00^{\prime} 33^{\prime \prime}$ (P81) lies between the two extended lobes of radio emission seen in the NVSS image (Fig. 3). The largest angular size measured from the edges of the radio source is close to about 500 arcsec, yielding a projected linear size of $\sim 1.3 \mathrm{Mpc}$ in a Universe with $q_{\mathrm{o}}=0.5$ and $H_{\mathrm{o}}=50 \mathrm{~km} \mathrm{~s}^{-1} \mathrm{Mpc}^{-1}$. This makes it a candidate giant radio galaxy (cf. Ishwara-Chandra \& Saikia 1999) with a somewhat unusual non-collinear structure. A higher resolution image would be useful to clarify the structure.
0039.0+3208 The position of the optical galaxy at RA(B1950) $00^{\mathrm{h}} 39^{\mathrm{m}} 04^{\mathrm{s}} .94$ and $\operatorname{Dec}(\mathrm{B} 1950) 32^{\circ} 08^{\prime} 27^{\prime \prime} \cdot 3$ (P81) is close to that of the brighter eastern lobe in the NVSS image (Fig. 3). The western and eastern radio components on opposite sides of the optical galaxy seen in the WSRT image by P81 are separated from the optical galaxy by $\sim 17.4$ and 14.4 arcsec respectively. The more extended emission on both sides seen in the NVSS image suggests that this is a candidate double-double radio galaxy (Schoenmakers et al. 2000) and merits further investigation.

0051.1+3058 The angular size has been estimated from the NVSS image (Fig. 3). The weaker NVSS component to the south-west may be related, but appears to be resolved out in the image by P81.

$\mathbf{0 1 1 0 . 0 + 3 1 5 2}$ The LAS given in Table 2 is between the prominent peaks seen in the image made by Wilkinson et al. (1998). A more extended halo is visible in a lower-resolution image (Hutchings et al. 1998).

0125.1+3229 The image resembles a single lobe in a doublelobed source. Two additional, weaker radio sources are visible to the south and east in the NVSS image (Fig. 3), but it is not clear if either of these is related to the B2 source.

0138.6+2927 The NVSS image (Fig. 3) shows a prominent component to the south-west. It is not clear if it is related to the source. The spectral indices $\alpha(327-1400 \mathrm{MHz})$ are $\sim 0.80$ and 0.85 for the source and the south-western component respectively.

0153.0+2941 The LAS given by P81 is listed. The NVSS image (Fig. 3) shows four components roughly symmetric about $\mathrm{RA}(\mathrm{B} 1950) 01^{\mathrm{h}} 53^{\mathrm{m}} 05^{\mathrm{s}}$ and $\operatorname{Dec}(\mathrm{B} 1950) 29^{\circ} 42^{\prime}$. A sensitive 

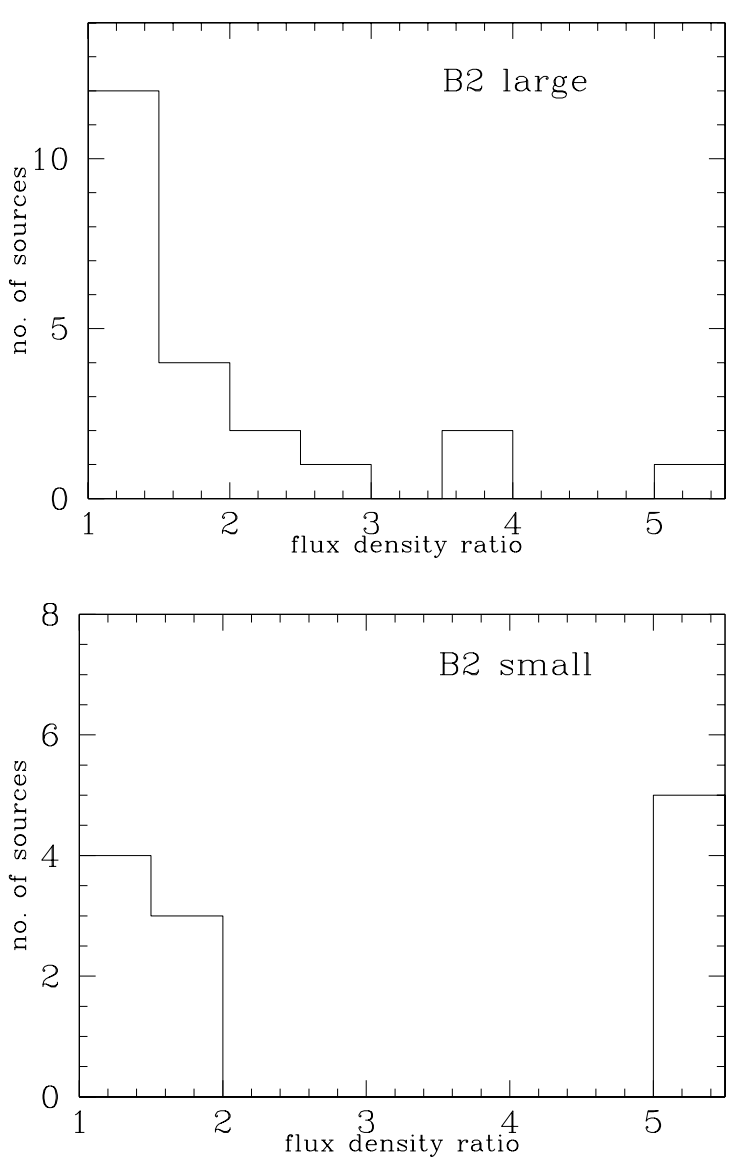

Fig. 4. The distributions of the flux density ratio, $R_{\mathrm{S}}$ for the $\mathrm{B} 2$ sources discussed in the text. All the sources with $R_{\mathrm{S}}>5$ have been placed in the last bin.

higher resolution image should help clarify any relationship between the components.

0204.6+3137 The VLBA image shows a core-jet structure oriented along a PA $\sim 135^{\circ}$ (Peck \& Taylor 2000). Our image suggests a strongly curved jet with the most distant component being along a $\mathrm{PA} \sim-90^{\circ}$.

0227.2+3206 P81 note that the source is resolved out in the WSRT observations. The source appears double-lobed in the NVSS image (Fig. 3), and the LAS has been estimated from this.

2330.5+2937 The source appears unresolved in our observations, although its spectral index, $\alpha_{408}^{5000} \sim 0.95$ (P81).

2342.5+2926 The spectral index between $327 \mathrm{MHz}$ and $5 \mathrm{GHz}$ is about 0.7 . It has a highly asymmetric radio structure, and has been detected in the 2MASXi survey (NED).

\section{Discussion}

Of the 19 sources observed by us, 13 have well-defined, double-lobed structures of which two also have detected central components. Of the remaining 6, two appear possibly double-lobed, two are single sources, one $(0204.6+3137)$ has a somewhat flatter radio spectrum and appears to have a corejet structure, while $0125.1+3229$ has a complex structure. The double-lobed radio sources tend to have Fanaroff-Riley class II structures with hot-spots at the outer edges of the sources.
Table 2. Properties of other sources in the sample.

\begin{tabular}{|c|c|c|c|c|c|c|}
\hline Source & $\mathrm{O}$ & $z$ & Str. & $\overline{F R}$ & LAS & Refs. \\
\hline $0003.0+3149$ & & & & & & $\mathrm{n}$ \\
\hline $0007.5+3028$ & & & $\mathrm{D} ?$ & & $57^{N}$ & \\
\hline $0007.8+3312$ & Q & 0.743 & $\mathrm{~T}$ & II & 78 & 1,2 \\
\hline $0008.7+3200$ & $\mathrm{G}$ & 0.1073 & D & II? & $357^{N}$ & $3,4, \mathrm{n}$ \\
\hline $0013.5+3222$ & & & $\mathrm{D}$ & II & 54 & 4 \\
\hline $0014.4+3152$ & Q & 1.086 & D & II & 17 & 4 \\
\hline $0015.9+3049$ & & & D & II & 49 & 4 \\
\hline $0039.0+3208$ & G & & D & II & $106^{N}$ & $\mathrm{n}$ \\
\hline $0039.9+2949$ & & & $\mathrm{D}$ & II & 12 & 4 \\
\hline $0051.1+3058$ & & & D & II? & $85^{N}$ & $\mathrm{n}$ \\
\hline $0055.0+3004$ & G & 0.0167 & $\mathrm{D}$ & II? & 3480 & 5,6 \\
\hline $0110.0+3152$ & Q & 0.6030 & $\mathrm{CJ} ?$ & & 0.7 & $7,8, \mathrm{n}$ \\
\hline $0110.6+2942$ & Q & 0.363 & $\mathrm{~T}$ & II & 76 & 9 \\
\hline $0138.6+2927$ & & & & & & $\mathrm{n}$ \\
\hline $0149.6+3335$ & Q & 2.4310 & $\mathrm{C}+\mathrm{E}$ & & 4 & 7,10 \\
\hline $0153.0+3001$ & & & $\mathrm{D}$ & & 14 & 4 \\
\hline $0153.0+2941$ & & & $\mathrm{D}$ & & 13 & $4, n$ \\
\hline $0154.3+3159$ & G & 0.0894 & HT & I & 102 & 11 \\
\hline $0157.8+3323$ & Q & & $\mathrm{T}$ & II & 25 & 4,12 \\
\hline $0200.8+3027$ & & & $\mathrm{C}+\mathrm{E}$ & & 69 & 4 \\
\hline $0202.1+3158$ & Q & 1.466 & $\mathrm{CJ}$ & & 0.04 & 13,14 \\
\hline $0206.6+3340$ & & & SR & & 9 & 16 \\
\hline $0216.8+3334$ & & & $\mathrm{D}$ & II & 12 & 17 \\
\hline $0217.8+3227$ & Q & 1.620 & $\mathrm{CJ} ?$ & & 3 & 7,18 \\
\hline $0222.3+3105$ & Q & 2.982 & $\mathrm{CJ} ?$ & & & 18 \\
\hline $0226.8+2924$ & & & $\mathrm{D}$ & II & 34 & 4 \\
\hline $0227.2+3206$ & & & $\mathrm{D}$ & II? & $97^{N}$ & $\mathrm{n}$ \\
\hline $2330.5+3038$ & & & SR & & $\sim 5$ & 4,15 \\
\hline $2335.0+3007$ & & & $\mathrm{D}$ & II? & 39 & 4 \\
\hline $2341.8+3019$ & & & $\mathrm{D}$ & II? & 19 & 4 \\
\hline $2347.8+3013$ & G & 0.374 & $\mathrm{D}$ & II? & 37 & $4,19,20,21$ \\
\hline $2348.5+3003$ & & & $\mathrm{~T}$ ? & II & 99 & 4 \\
\hline $2349.8+3247$ & Q & 0.659 & $\mathrm{~T}$ & II & 60 & $1,22,23$ \\
\hline
\end{tabular}

References: 1: Price et al. (1993); 2: Wardle \& Potash (1985); 3: NED; 4: Padrielli et al. (1981); 5: Bridle et al. (1976); 6: Willis et al. (1981); 7: Wilkinson et al. (1998); 8: Hutchings et al. (1998); 9: Garrington et al. (1991); 10: Neff \& Hutchings (1990); 11: Owen \& Ledlow (1997); 12: Minter \& Spangler (1996); 13: Fey \& Charlot (2000); 14: Fomalont et al. (2000); 15: Rudnick \& Adams (1979); 16: Fanti \& Padrielli (1977); 17: Röttgering et al. (1994); 18: Willott et al. (1998); 19: Zirbel (1997); 20: Nilsson et al. (1993); 21: Grueff et al. (1981); 22: Potash \& Wardle (1980); 23: Bogers et al. (1994).

Unfortunately, the optical identification and redshift information for this sample is still very incomplete for investigating in detail the source properties and all symmetry parameters. Further, radio cores have been detected in only a small fraction of the sources. Therefore, we examine only the flux density ratio, $R_{\mathrm{S}}$, of opposing lobes of the steep-spectrum double-lobed sources, classified as D or T in Tables 1 and 2. This parameter can be used to probe asymmetries in the gas distribution in the central regions of these galaxies, which might be related to the infall of gas which fuels the radio source. The radio jet propagating outwards on the denser side will be more dissipative, and hence should be brighter, and closer to the nucleus (cf. Eilek \& Shore 1989; Gopal-krishna \& Wiita 1991). Although 

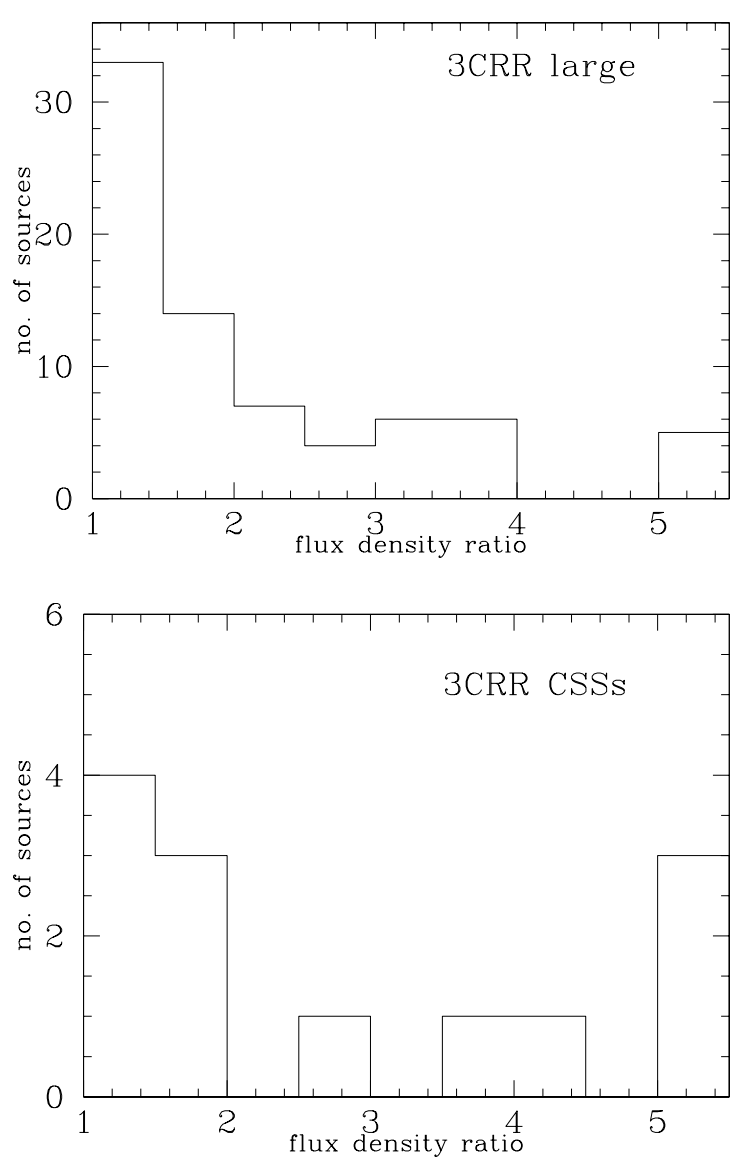

Fig. 5. The distributions of the flux density ratio, $R_{\mathrm{S}}$ for the 3CRR sources discussed in the text. All the sources with $R_{\mathrm{s}}>5$ have been placed in the last bin.

relativistic motion of the lobes could also affect their brightness asymmetry, this alone is not likely to generate highly asymmetric sources since the velocity of advancement is expected to be only mildly relativistic, and most of our objects are likely to be inclined at large angles to the line of sight. Also, while the hotspots advance outwards, the rest of the radio lobe emission is due to backflow from these high-brightness features. For a velocity of advancement of $\sim 0.1$ c (cf. Scheuer 1995), the brightness asymmetry of the lobes for a source inclined at $30^{\circ}$ to the line of sight is $\lesssim 2$. Nevertheless, for a few quasars, the brightness asymmetry may be enhanced due to relativistic motion of the hotspots. However, this is not likely to be an important factor for the objects in our sample because in addition to the low velocities, these are lobe-dominated objects and are unlikely to be inclined at small angles to the line of sight. Also, most of the objects ( $\sim 75$ per cent) are not identified with quasars, and are therefore expected to be inclined at $>45^{\circ}$ to the line of sight, i.e. the dividing line between radio galaxies and quasars in the unified scheme (Barthel 1989), thereby further decreasing the expected asymmetry due to the velocity of advancement.

Considering the steep-spectrum, double-lobed B2 sources, we define the "small-source" sample to consist of those with an LAS less than 10 arcsec. The remaining objects constitute the "large-source" sample. The median angular size of the sample of small sources is about 4 arcsec compared with about 40 arcsec for the larger sources. The ratio of flux density of the oppositely directed lobes, $R_{\mathrm{s}}$, has been estimated for all these sources from either our observations or the references listed in Table $2 . R_{\mathrm{s}}$ ranges from $\sim 1.4$ to 20 for the small-source sample, and has a median value of about 2 . For the large-source sample, $R_{\mathrm{S}}$ ranges from $\sim 1.0$ to 7 , with a median value of $\sim 1.4$ (Fig. 4).

It can be seen from Fig. 4 that a significant fraction of the small sources show large asymmetries. Defining a very asymmetric source to be one with $R_{\mathrm{s}}>5$, we find that 5 of the 12 small-sized objects ( $\sim 40$ per cent) are highly asymmetric, while only one of the $22(\sim 5$ per cent $)$ objects in the large-source $\mathrm{B} 2$ sample have $R_{\mathrm{S}}>5$. It is of interest to compare this with earlier studies of the flux density ratio for sources in the well-known 3CRR sample (e.g. Saikia et al. 1995, 2001; Arshakian \& Longair 2000). For example, for the 3CRR sources studied by Saikia et al,, which was confined to the high-luminosity FRII sources, the median values of $R_{\mathrm{S}}$ for the large sources and the CSSs are about 1.8 and 2 respectively. This is similar to that of the B2 sources. The distribution for the CSSs again appears to have a number of very asymmetric sources with 3 of the 13 sources (about 23 per cent) having a value of $R_{\mathrm{S}}>5$, while only 5 of the 75 ( $\sim 7$ per cent) non-CSS 3CR sources exhibit such a high degree of asymmetry (Fig. 5). The small-sized sources in both the B2 and 3CRR samples appear to have a greater fraction of objects exhibiting high flux-density asymmetry compared with the larger sources. Excluding these highly asymmetric CSS objects, the remainder of the population of CSS sources show very similar brightness ratios to those of the larger objects.

We have also compared this result for the CSS objects with the large sample of CSSs selected from the B3-VLA sample with $S(408) \geq 0.8$ Jy (Fanti et al. 2001). Fanti et al. have classified all sources with two distinct steep-spectrum $(\alpha \gtrsim 0.5)$ components as double, and those with three distinct steepspectrum components as triple. We consider all the doubles, plus just one triple source, $0744+464$. This source is collinear and the central feature, which has a flatter spectrum, contributes less than a few per cent of the total flux density and does not significantly affect the flux density ratio. The $R_{\mathrm{S}}$ distribution for the 39 sources from this sample is shown in Fig. 6. The median value of $R_{\mathrm{S}}$ for the B3-VLA CSS sample is about 2.4, with 8 of the 39 objects ( $\sim 21$ per cent) having a value of $R_{\mathrm{s}}>5$. This is consistent with the results for the B2 and 3CRR samples.

To assess the statistical significance of the results, we have considered all the CSS sources from the B2, 3CRR and B3VLA samples together and compared their $R_{\mathrm{S}}$ distribution with that of the larger sources from the B2 and 3CRR samples. This increases the sample sizes to a total of 64 CSS objects and 97 larger sources, and should yield a statistically reliable result. Considering the above samples, the median values of $R_{\mathrm{S}}$ for the CSS objects and the larger sources are about 2 and 1.5 respectively. A Kolmogorov-Smirnov test shows the two distributions to be different at a confidence level of greater than 99 per cent. However, if we exclude the most asymmetric objects, i.e. those with $R_{\mathrm{S}}>5$, the distributions for the rest of the sources are not significantly different. The fraction of such 


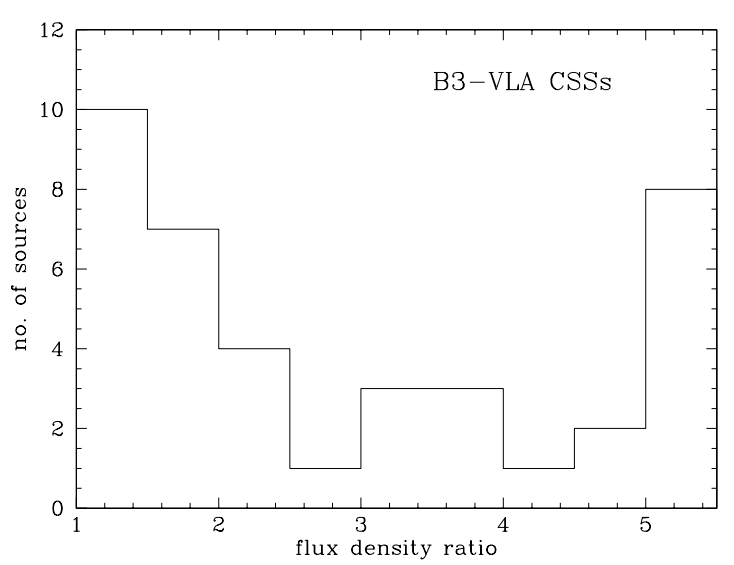

Fig. 6. The distributions of the flux density ratio, $R_{\mathrm{S}}$ for the B3-VLA CSS sources discussed in the text. All the sources with $R_{\mathrm{s}}>5$ have been placed in the last bin.

asymmetric sources for the combined sample is 25 per cent for the CSSs and 6 per cent for the larger sources. If the threshold for defining a very asymmetric source is changed to $R_{\mathrm{s}}>4$, the fraction for the CSS objects increases to about 30 per cent, while that for the larger objects remains the same. These trends are also seen if the confirmed quasars and the remaining objects are considered separately.

Thus, in about 25 per cent of the CSSs the flux density asymmetry is significantly increased, possibly due to interaction of one of the jets with a much denser external medium or a dense cloud on one side of the nucleus. These density asymmetries might be intimately related to the infall of gas which fuels the radio source. Taking the typical distances of these clouds of gas from the nucleus of the galaxy to be about 5 to $10 \mathrm{kpc}$, the fraction of very asymmetric sources found above suggests that the size of the "clouds" should be about 3 to $7 \mathrm{kpc}$. This is typical of the sizes of dwarf galaxies (cf. Swaters 1999).

It is also of interest to enquire whether the brightness asymmetries of CSSs depend on cosmic epoch because of the larger incidence of interactions and mergers in the past, as seen in Hubble Space Telescope studies of distant galaxies (cf. Abraham et al. 1996; Brinchmann et al. 1998; Ellis et al. 2000). For this, we have considered the double-lobed CSSs with a measured or estimated redshift from the combined sample. The $R_{\mathrm{s}}$-redshift diagram for these 43 sources is presented in Fig. 7. It is quite clear from the figure that the flux density asymmetry shows no significant dependence on redshift. This suggests that although interactions and mergers may increase with redshift globally, the density asymmetries in these CSS objects which are young and possibly still being fuelled by the infall of gas, are similar at different redshifts.

\section{Conclusions}

Radio images of small sources from the B2 sample of intermediate strength have been presented. About 25 per cent of the CSSs from the B2, 3CRR and the B3-VLA samples exhibit a higher degree of brightness asymmetry, with the flux density ratio $R_{\mathrm{S}} \gtrsim 5$. The fraction of such highly asymmetric objects amongst the larger sources is only about 6 per cent.

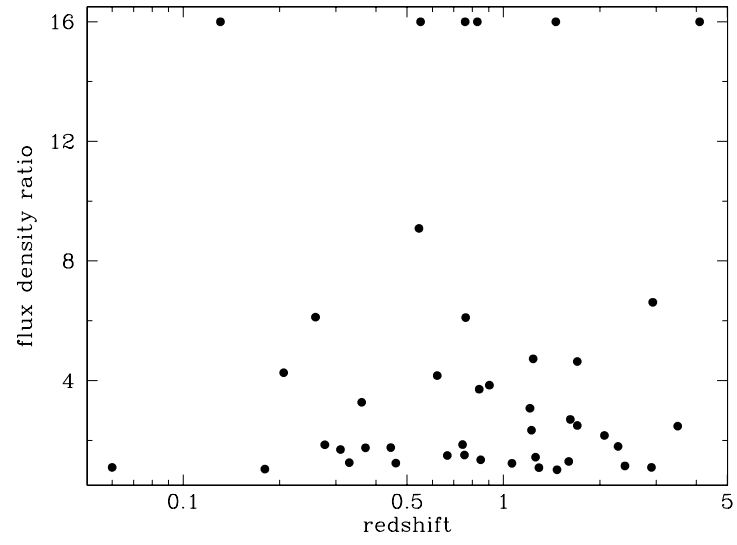

Fig. 7. The $R_{\mathrm{s}}$-redshift diagram for the CSSs in the 3CRR, B2 and B3-VLA samples discussed in the text. The six sources at the upper part of the figure have $R_{\mathrm{s}}>16$.

The larger fraction of highly asymmetric objects amongst CSS sources might be due to interactions of the radio plasma with infalling clouds of gas which fuel the radio source. The size of such a cloud is estimated to be about a few kpc, which is typical of dwarf galaxies. The brightness asymmetry for these objects shows no significant dependence on redshift, although such a situation might be expected due to the larger incidence of interactions and mergers in the past. This suggests that the density asymmetries in these young radio sources are similar at different redshifts.

Acknowledgements. We thank the referee, Ignas Snellen, for several constructive comments which considerably improved the paper, and also for reminding us of the paper by Carla Fanti and her collaborators on the B3-VLA sample. The National Radio Astronomy Observatory is a facility of the National Science Foundation operated under cooperative agreement by Associated Universities, Inc. We have made use of the NASA/IPAC Extragalactic Database (NED), which is operated by the Jet Propulsion Laboratory, California Institute of Technology under contract with the National Aeronautics and Space Administration. We thank Dave Rossi for preserving the 9-track tape containing the calibrated data in his loft for many years, and Dave Shone for managing to read the tape! One of us (DJS) would like to thank the PPARC Visitors Programme at Jodrell Bank Observatory and Ralph Spencer who looks after this programme, for financial support, and Peter Thomasson for hospitality while most of this work was done.

\section{References}

Abraham, R. G., Tanvir, N. R., Santiago, B. X., et al. 1996, MNRAS, 279, L47

Akujor, C. E., \& Garrington, S. T. 1995, A\&AS, 112, 235

Arshakian, T. G., \& Longair, M. S. 2000, MNRAS, 311, 846

Baars, J. W. M., Genzel, R., Pauliny-Toth, I. I. K., \& Witzel, A. 1977, A\&A, 61, 99

Barthel, P. D. 1989, ApJ, 336, 606

Bogers, W. J., Hes, R., Barthel, P. D., \& Zensus, J. A. 1994, A\&AS, 105, 91

Bridle, A. H., Davis, M. M., Meloy, D. A., et al. 1976, Nature, 262, 179

Brinchmann, J., Abraham, R., Schade, D., et al. 1998, ApJ, 499, 112

Carilli, C. L., Menten, K. M., Reid, M. J., Rupen, M. P., \& Min, S. Y. 1998, ApJ, 494, 175 
Colla, G., Fanti, C., Fanti, R., et al. 1970, A\&AS, 1, 281

Condon, J. J., Cotton, W. D., Greisen, E. W., et al. 1998, AJ, 115, 1693

Dunlop, J. S., \& Peacock, J. A. 1990, MNRAS, 247, 19

Eilek, J. A., \& Shore, S. N. 1989, ApJ, 342, 187

Ellis, R. S., Abraham, R. G., Brinchmann, J., \& Menanteau, F. 2000, A\&G, No. 2,10

Fanti, R., \& Padrielli, L. 1977, A\&AS, 29, 263

Fanti, C., Fanti, R., Dallacasa, D., et al. 1995, A\&A, 302, 317

Fanti, C., Pozzi, F., Dallacasa, D., et al. 2001, A\&A, 369, 380

Fey, A. L., \& Charlot, P. 2000, ApJS, 128, 17

Fomalont, E. B., Frey, S., Paragi, Z., et al. 2000, ApJS, 131, 95

Garrington, S. T., Conway, R. G., \& Leahy, J. P. 1991, MNRAS, 250, 171

Gopal-krishna, \& Wiita, P. J. 1991, ApJ, 373, 325

Grueff, G., Schiavo-Campo, P., Tanzella-Nitti, G., Vigotti, M., \& Kotanyi, C. 1981, A\&AS, 44, 241

Hutchings, J. B., Dewey, A., \& Chaytor, D. 1998, PASP, 110, 111

Ishwara-Chandra, C. H., \& Saikia, D. J. 1999, MNRAS, 309, 100

Jeyakumar, S., \& Saikia, D. J. 2000, MNRAS, 311, 397

Junor, W., Salter, C. J., Saikia, D. J., Mantovani, F. M., \& Peck, A. B. 1999, MNRAS, 308, 955

Kapahi, V. K. 1981, A\&AS, 43, 381

Kapahi, V. K., Athreya, R. M., Subrahmanya, C. R., et al. 1998a, ApJS, 118, 327

Kapahi, V. K., Athreya, R. M., van Breugel, W., McCarthy, P. J., \& Subrahmanya, C. R. 1998b, ApJS, 118, 275

Kunert, M., Marecki, A., Spencer, R. E., Kus, A. J., \& Niezgoda, J. 2002, A\&A, in press

Laing, R. A., Riley, J. M., \& Longair, M. S. 1983, MNRAS, 204, 151

Large, M. I., Mills, B. Y., Little, A. G., Crawford, D. F., \& Sutton, J. M. 1981, MNRAS, 194, 693

Mantovani, F., Junor, W., Fanti, R., Padrielli, L., \& Saikia, D. J. 1994, A\&A, 292, 59

Minter, A. H., \& Spangler, S. R. 1996, ApJ, 458, 194

Morganti, R., Oosterloo, T. A., Tadhunter, C. A., et al. 2001, MNRAS, 323, 331

NED (http://nedwww.ipac. caltech. edu)

Neff, S. G., \& Hutchings, J. B. 1990, AJ, 100, 1441

Nilsson, K., Valtonen, M. J., Kotilainen, J., \& Jaakkola, T. 1993, ApJ, 413, 453

O’Dea, C. P. 1998, PASP, 110, 493

Owen, F. N., \& Ledlow, M. J. 1997, ApJS, 108, 41

Owsianik, I., \& Conway, J. E. 1998, A\&A, 337, 69
Padrielli, L., Kapahi, V. K., \& Katgert-Merkelijn, J. K. 1981, A\&AS, 46, 473

Pauliny-Toth, I. I. K., Witzel, A., Preuss, E., et al. 1978, AJ, 83, 451

Peck, A. B., \& Taylor, G. B. 2000, ApJ, 534, 90

Peck, A. B., Taylor, G. B., Fassnacht, C. D., Readhead, A. C. S., \& Vermeulen, R. C. 2000, ApJ, 534, 104

Potash, R. I., \& Wardle, J. F. C. 1980, ApJ, 239, 42

Price, R., Gower, A. C., Hutchings, J. B., et al. 1993, ApJS, 86, 365

Readhead, A. C. S., Taylor, G. B., Xu, W., et al. 1996a, ApJ, 460, 612

Readhead, A. C. S., Taylor, G. B., Pearson, T. J., \& Wilkinson, P. N. 1996b, ApJ, 460, 634

Rengelink, R. B., Tang, Y., de Bruyn, A. G., et al. 1997, A\&AS, 124, 259

Röttgering, H. J. A., Lacy, M., Miley, G. K., Chambers, K. C., \& Saunders, R. 1994, A\&AS, 108, 79

Rudnick, L., \& Adams, M. T. 1979, AJ, 84, 437

Saikia, D. J., Swarup, G., \& Kodali, P. D. 1985, MNRAS, 216, 385

Saikia, D. J., Singal, A. K., \& Cornwell, T. J. 1987, MNRAS, 224, 379

Saikia, D. J., Jeyakumar, S., Wiita, P. J., Sanghera, H. S., \& Spencer, R. E. 1995, MNRAS, 276, 1215

Saikia, D. J., Jeyakumar, S., Salter, C. J., et al. 2001, MNRAS, 321, 37

Scheuer, P. A. G. 1995, MNRAS, 277, 331

Schilizzi, R. T., Tschager, W., Snellen, I. A. G., et al. 2000, AdSpR, 26, 709

Schoenmakers, A. P., de Bruyn, A. G., Röttgering, H. J. A., van der Laan, H., \& Kaiser, C. R. 2000, MNRAS, 315, 371

Spencer, R. E., Schilizzi, R. T., Fanti, C., et al. 1991, MNRAS, 250, 225

Stickel, M., \& Kühr, H. 1994, A\&AS, 103, 339

Swaters, R. A. 1999, Ph.D. Thesis, Rijksuniversiteit Groningen

van Breugel, W. J. M., Fanti, C., Fanti, R., et al. 1992, A\&A, 256, 56

Wall, J. V., \& Peacock, J. A. 1985, MNRAS, 216, 173

Wardle, J. F. C., \& Potash, R. I. 1985, Physics of Energy Transport in Extragalactic Radio Sources, ed. A. H. Bridle, \& J. A. Eilek, NRAO Workshop No. 9, 30

Warwick, R. S. 1977, MNRAS, 179, 1

Wilkinson, P. N., Browne, I. W. A., Patnaik, A. R., Wrobel, J. M., \& Sorathia, B. 1998, MNRAS, 300, 790

Willis, A. G., Strom, R. G., Bridle, A. H., \& Fomalont, E. B. 1981, A\&A, 95, 250

Willott, C. J., Rawlings, S., Blundell, K. M., \& Lacy, M. 1998, MNRAS, 300, 625

Zirbel, E. L. 1997, ApJ, 476, 489 